\title{
Of mice and men
}

\author{
We need a systematic way to formulate and tackle problems in nanotoxicology, and to compare and \\ combine the results from individual studies.
}

When a foreign material enters the body, our immune system detects, isolates and destroys it in a number of ways. Some of these tasks are performed by specialized cells called macrophages, which engulf and neutralize the material in a process called phagocytosis, but if this fails, the foreign material can cause inflammation and other problems. The emergence of new properties in nanoparticles, which are not found in bulk materials, has led to numerous calls for further research into the toxicity of engineered nanomaterials, but the scale of the challenge is enormous. Recently it was estimated that it could take between 34 and 53 years, and cost between $\$ 249$ million and $\$ 1.18$ billion dollars, to perform toxicity tests on existing nanomaterials in just the United States ${ }^{1}$.

\section{There is a shortage of data about the exposure of workers to nanomaterials.}

Testing for toxicity involves a number of considerations: the duration and route of exposure (which could be by inhalation, ingestion, injection or skin absorption), the dosage, the formulation of the test material, the reference and standard materials used for comparisons, and the questions of which animal model to use and which end points to measure. Testing nanomaterials for toxicity brings a new dimension to the problem because traditional methods may not work. Moreover, nanomaterials can stimulate or suppress the immune response $^{2}$, and the consequences of this remain unknown.

When testing for toxicity the experimental design depends on whether the nanomaterials enter the body accidentally (as in workplace or environmental exposure) or are deliberately introduced (as in new medicines). In medicine, toxicology testing is important because it tells us whether the risks of a new material for treatment outweigh the benefits. In other areas, these tests can warn us if exposures are likely to be a threat to our health or the environment (see, for example, the Letter by John Ferry and co-workers on page 441 of this issue).

Early studies, in which carbon nanotubes were delivered into the lungs of mice through the windpipe (a procedure called instillation) showed inflammation in the lungs and high animal mortality because the nanotubes clogged up the upper airways of the mice ${ }^{3,4}$. Inhalation studies, which involve exposing the animals to aerosols of the material in a chamber over time, are more representative of the natural route of entry when considering occupational hazards. However, the levels of exposure in these inhalation experiments, and their relevance to the concentrations that are found in the workplace, remain debatable. Nonetheless, all studies should provide rough estimates that translate individual findings in animals into approximate exposure levels for humans so that the results can be put into perspective. Moreover, doing so would also lead to improvements in the design of future studies.

Building on previous work ${ }^{5}$, in which they showed that mice inhaling low levels of multiwalled carbon nanotubes can have a suppressed immune system, Jacob McDonald and colleagues report on page 451 of this issue that this suppression originates from signals in the lung that activate enzymes in the spleen. As Alison Elder comments in an accompanying News \& Views (on page 409 of this issue), this study is significant because it uses the inhalation model to achieve physiologically relevant exposures. Moreover, the suppression of the immune function caused by repeated exposures to nanotubes highlights the potential health risks faced by workers in factories making these materials (assuming that studies in mice are relevant to humans). Although the shortage of data about the exposure of workers to nanomaterials makes it difficult to place such studies into a proper context, Elder encourages the inclusion of inhalation studies in the framework for characterization of hazards.

Elsewhere, on page 411 of this issue, Marina Dobrovolskaia and colleagues outline how traditional methods can produce unreliable results when used to evaluate the immune response of nanoparticles. Drawing on discussions at an international workshop on this topic, they discuss what can be done until new methods are developed. Although in vitro tests are becoming popular alternatives to animal studies, no such test has yet been validated as a substitute for animal studies, and using a combination of tests would, at present, seem to be the most reasonable approach from the regulation point of view.

\section{Comparing new medicines to approved formulations should be standard practice.}

The message is loud and clear: the pace of nanotoxicology research needs to be increased to reflect the growing use of nanomaterials in commercial products. However, this needs to happen in a systematic way so that priorities can be set based on what we have learnt so far, and then revised in the light of new knowledge. We need good approaches (be they computational models ${ }^{6}$ or field studies) to forecast the levels and types of exposures, and to design experiments that resemble the real conditions and settings, and we also need new tools to evaluate these experiments. New nanomedicines demand new toxicology models to determine risk-to-benefit ratios, and it should also become standard practice to make direct comparisons with well-established alternatives. Finally, a standardized set of methods, nomenclature and testing requirements is needed so that data from different laboratories can be compared. Achieving all this will only be possible if all parties - governments, companies and the research community - share the responsibility and work together.

\footnotetext{
References

1. Choi, J.-Y., Ramachandran, G. \& Kandlikar, M. Environ. Sci. Tech 43, 3030-3034 (2009).

2. Dobrovolskaia, M. \& McNeil, S. Nature Nanotech. 2, 469-478 (2007).

3. Warheit, D. B. et al. Toxicol. Sci. 77, 117-125 (2004)

4. Lam, C. W., James, J. T., McCluskey, R. \& Hunter, R. L. Toxicol. Sci. 77, 126-134 (2004).

5. Mitchell, L. A. et al. Toxicol. Sci. 100, 203-214 (2007).

6. Barnard, A. et al. Nature Nanotech. 4, 332-335 (2009).
} 